\title{
Genauigkeit moderner Kraftmessdosen- Stand der Technik und Anwendungen
}

\author{
Thomas Kleckers \\ Hottinger Baldwin Messtechnik GmbH \\ Product Marketing \\ Im Tiefen See 45 \\ 64293 Darmstadt \\ Thomas.kleckers@hbm.com
}

\section{Einführung}

Moderne Kraftsensoren wie die S9M von HBM erreichen höchste Genauigkeiten. Linearitätsfehler, relative Umkehrspanne und Temperatureinflüsse sind kleiner als $0,02 \%$ relativ zum Endwert. Diese Eigenschaften eröffnen neue Anwendungen, bei denen die Genauigkeit weniger im Mittelpunkt steht, als vielmehr die Wirtschaftlichkeit und die Zuverlässigkeit einer Kraftmessung.

\section{Kraftaufnehmer auf Basis von Dehnungsmessstreifen}

Kraftaufnehmer auf Basis von Dehnungsmessstreifen verfügen über einen so genannten Federkörper oder Verformungskörper, in dem die zu messenden Kräfte eingeleitet werden.

Dabei verformt sich der Federkörper und an der Oberfläche entstehen Dehnungen. Die Aufgabe des Federkörpers ist es also, die zu messende Kräfte möglichst wiederholbar und linear in Dehnungen umzuwandeln. Mit Auswahl von Material und Design eines Federkörpers legt man viele Eigenschaften eines Kraftaufnehmers fest.

Das eigentliche Sensorelement ist der Dehnungsmessstreifen. Diese Elemente bestehen aus einer Isolierschicht, dem so genannten Träger, auf dem ein Messgitter aufgebracht ist. Solche Dehnungsmessstreifen werden an geeigneter Stelle auf den Federkörper geklebt. Dabei werden in der Regel vier Dehnungsmessstreifen verwendet, von denen zwei so installiert werden, dass sie unter Einwirkung einer Kraft gedehnt werden, zwei werden gestaucht.

Diese vier DMS werden in der Wheatstonschen Brückenschaltung verschaltet. Wie in der Zeichnung unten gezeigt, wird die Wheatstonbrücke mit einer Speisespannung versorgt. Eine Ausgangsspannung entsteht immer dann, wenn die vier Widerstände unterschiedlich sind: z.B. wenn sich der Widerstand der DMS durch Dehnung ändert.

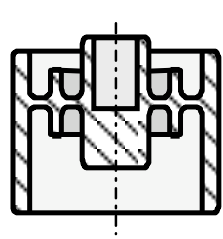

Federkörper

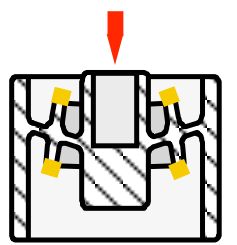

Die zu messende Kraft bewirkt Verformungen des Federkörpers. Dehnungsmessstreifen wandeln die Dehnungen in Widerstandsänderungen um
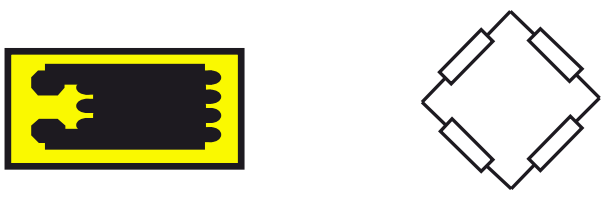

Mit Hilfe der Wheatstone -Brücke können kleinste

Widerstandsänderungen als elektrische Spannungen gemessen werden.

Arbeitsweise eines Kraftaufnehmers auf Basis von Dehnungsmessstreifen am Beispiel eines Ringtorsionsaufnehmers (Type: C18)

Das Ausgangssignal hängt von der Widerstandsänderung der DMS und damit dirékt von der eingeleiteten Kraft ab. 
Dieses Prinzip ist millionenfach bewährt und bietet Zahlreiche Vorzüge. Die wichtigsten:

- Ändern sich die elektrischen Widerstände der DMS in gleicher Richtung mit gleichem Betrag, so wird keine Ausgangsspannung erzeugt. Somit können viele parasitäre Einflüsse, wie z.B. Temperaturabhängigkeit des Nullpunktes, Biegemomenteinflüsse, Querkrafteinflüsse kompensiert werden (siehe unten)

- Das Messprinzip erlaubt die Herstellung von Kraftaufnehmern mit sehr hohen Genauigkeiten bei vergleichsweise geringen Kosten

- Die Nennkraft des Aufnehmers wird nur durch die Steifigkeit des Federköpers bestimmt. Bei HBM stehen Aufnehmer mit Nennkräften zwischen $10 \mathrm{~N}$ und $5 \mathrm{MN}$ zur Verfügung

Insbesondere der erste Punkt ist von Bedeutung. Es zeigt sich, dass durch Einsatz der Wheatstonschen Brückenschaltung viele ungewollte Einflüsse kompensiert werden.

Insbesondere der Einfluss der Temperatur auf den Nullpunkt (TKNull) kompensieren sich zu einem sehr guten Grad aus. Jeder Dehnungsmessstreifen zeigt bei Temperaturänderung ein Ausgangssignal, die so genannte scheinbare Dehnung.

\section{temperature variation LK11G-6/350}

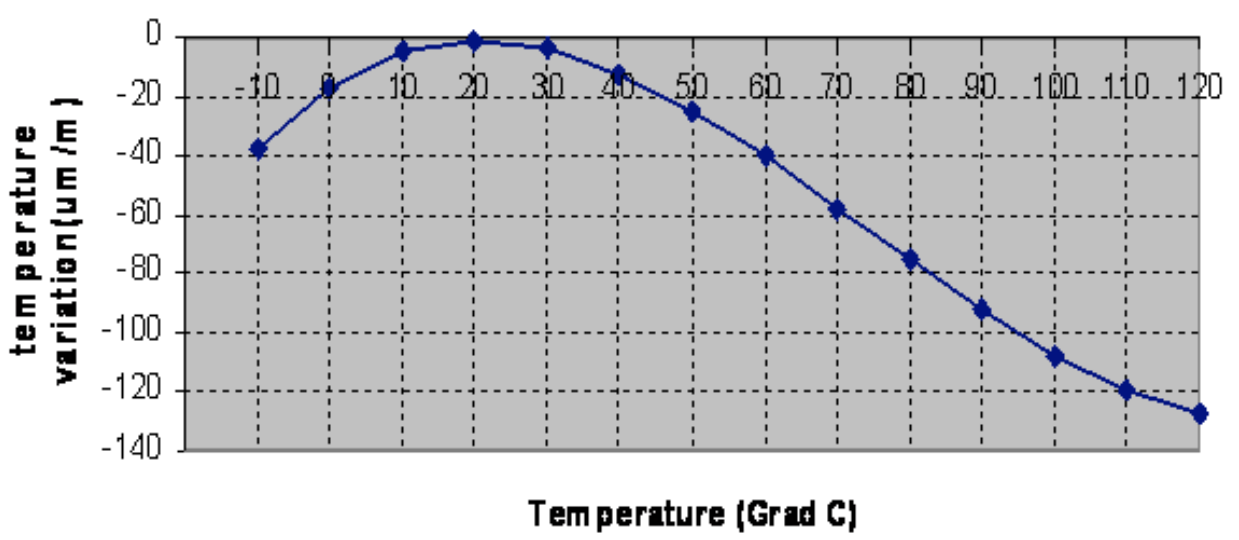

Bild 2: Ausgangssignals eines DMS in Abhängigkeit von der Temperatur

Die Kompensationswirkung ergibt sich aus der Tatsache, dass alle vier DMS bei Temperaturänderung hinsichtlich der Richtung und des Betrages der Widerstandsänderung gleich verhalten. Da zwei DMS in der Gleichung positiv und zwei negativ eingehen, ergibt sich bei Temperaturänderung insgesamt nahezu kein Ausgangssignal.

Der verbleibende, kleine Restfehler kann durch spezielle Nickelelemente, die in die Wheatsonsche Brücke geschaltet werden, korrigiert.

Durch zuschalten von temperaturabhängigen Widerständen (ebenfalls Nickelelemente) in die Speiseleitung lässt sich die Temperaturabhängigkeit des Kennwertes (TKC) abgleichen. Bei Temperaturänderung verringert sich das E - Modul der Werkstoffe, wodurch bei gleicher Krafteinwirkung mehr Dehnung entsteht. Zusätzlich ist die Empfindlichkeit der DMS von der Temperatur abhängig. Die Widerstände in der Speiseleitung kompensieren dies, in dem die Widerstandserhöhung bei höherer Temperatur zu einem größeren Spannungsabfall führt, hierdurch verringert sich die Spannung an der Wheatsonschen Brücke, in Folge sinkt das Ausgangssignal.

Linearitäsfehler entstehen durch sich unter Last ändernde geometrische Verhältnisse. Kraftaufnehmer lassen sich durch geschickte Auswahl des Designs des Federkörpers und durch exaktes Positionieren der Dehungsmesstreifen zu sehr guten Linearitäten optimieren

In Bild 3 wird eine Zusammenfassung der Kompensationsmöglichkeiten gegeben. Neben den oben detailierter beschriebenen Einflüssen TKnull und TKC ist es ohne weiteres möglich, die Linearität abzugleichen, sowie einen gewünschten Kennwert durch Abgleich zu erreichen. 


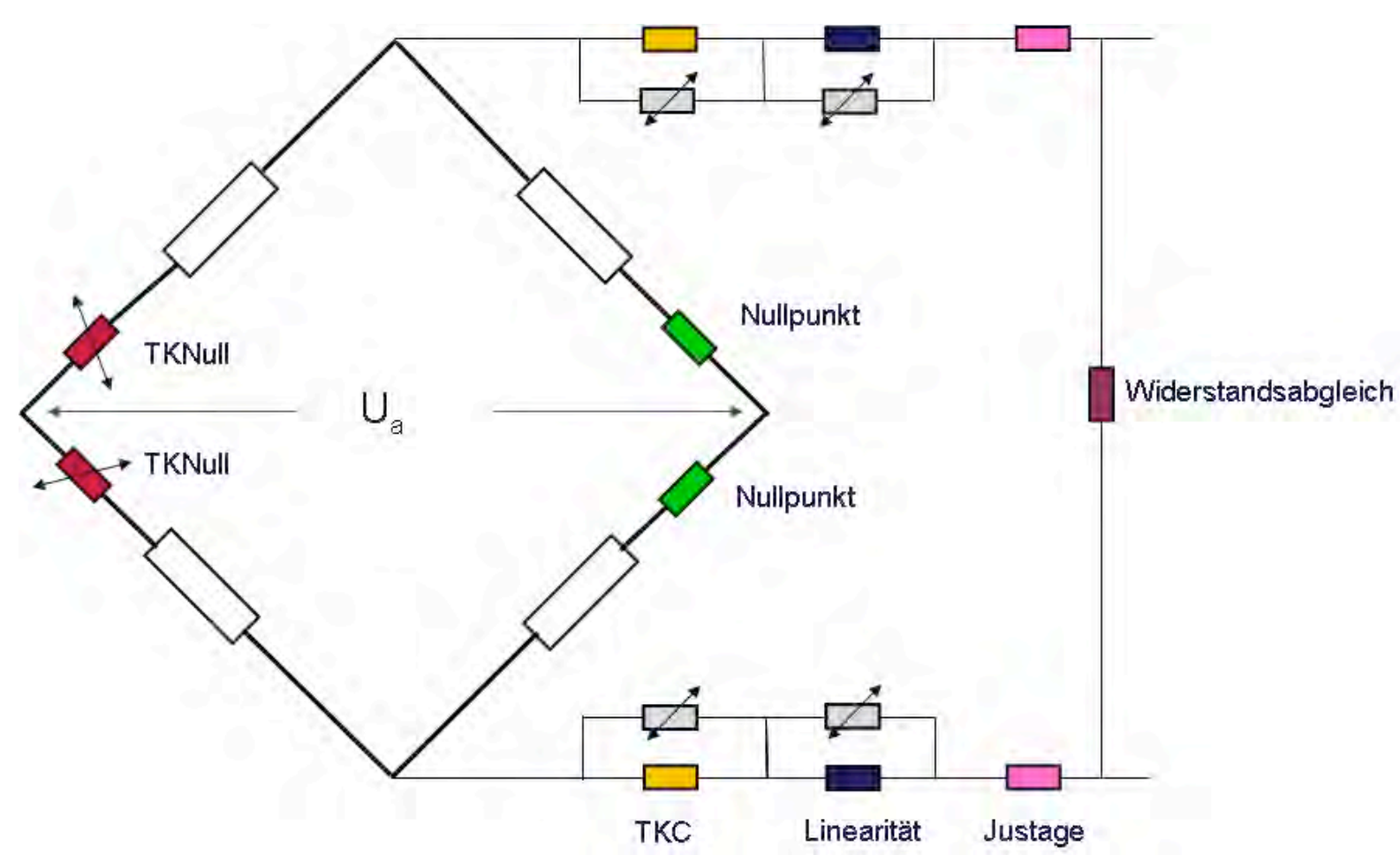

Bild 2: Verschaltung eines Kraftaufnehmers mit TKNull- und TKC - Abgleich. Zusätzlich Abgleichelemente für Kennwert und Linearität

Die Technologie der DMS erlaubt darüber hinaus auch die Kompensation von mechanischen Einflüssen, wie z.B. Biegemomenten oder Querkräften, die nicht erfasst werden sollen.

\section{Zur Fehlerbetrachtung von Messungen mit Kraftaufnehmern}

Bei Messung mit Kraftaufnehmern auf Basis von DMS unterscheidet man zwei Fehlergruppen: Fehler, die unabhängig von der anliegenden Kraft ein bestimmtes Ausgangssignal erzeugen, und Fehler, deren Größe der im Moment der Betrachtung anliegenden Kraft proportional sind.

Der Temperatureinfluss auf den Nullpunkt ist ein Beispiel für einen lastunabhängigen Fehler: Diese Messungenauigkeit weist einen bestimmten Betrag auf, der unabhängig von der gemessenen Kraft ist. Betrachtet man einen solchen Fehler relativ zum Ausgangsignal, so zeigt sich, dass der Einfluss des TkNull immer dann besonders groß ist, wenn nur ein kleiner Teil der Nennkraft benutzt wird. Der Betrag ist immer gleich, jedoch steigt auf Grund des kleinen Nutzsignals in dieser Situation der relative Anteil. Neben dem TKNull ist auch der Linearitätsfehler auf den Endwert bezogen

Fehler, die relativ zum Istwert sind (Istwertbezogene Fehler) werden relativ zum aktuell anliegenden Signal berechnet. Hierunter fällt zum Beispiel die Temperaturabhängigkeit des Kennwertes (TKC), das Kriechen oder auch die Toleranz einer eventuell durchgeführten Kalibrierung.

Bei einer Fehlerbetrachtung werden die Einzelfehler geometrisch addiert, d.h. eine signifikante Verbesserung der Messgenauigkeit lässt sich nur erreichen, wenn die größten Einzelfehler verbessert werden. In vielen Fällen ist der TKNull und die Linearität von entscheidender Bedeutung. Da diese Fehler auf den Endwert, d.h. auf das Ausgangssignal bei voller Ausnutzung der Nennkraft bezogen werden, ist eine Verbesserung dieser Paramter besonders effektiv. und erlaubt einen Einsatz des Kraftaufnehmers auch im so genannten Teillastbereich, d.h. eine Ausnutzung nur eines Teilbereichs der Nennkraft.

Der Kraftaufnehmer S9M von HBM bietet einen TKNull und eine Linearität von nur 200 ppm relativ zum Endwert. Wird ein solcher Kraftaufnehmer bei $20 \%$ der Nennkraft eingesetzt, so liegt der Fehler, den Linearität bzw. der TKNull relativ zur anliegenden Kraft verursachen nur bei $0,1 \%$. 
Bei größeren Nennkräften bieten sich die so genannten Pancakes an, die eine relative Umkehrspanne ab $300 \mathrm{ppm}$ relative zur Nennkraft aufweisen, und wegen Ihrer außerordentlich günstigen DMS - Anordnung einen TKNull von nur 150 ppm zeigen.

\section{Die Vorzüge der Genauigkeit}

Es ist leicht ersichtlich, dass die hohe Genauigkeit des Aufnehmers erst den Einsatz bei kleineren Kräften ermöglicht. Dies kann unter verschiedenen Gesichtspunkten eingesetzt werden:

- Die Messkette kann für hohe Überlasten ausgelegt werde, z.B. um eine Beschädigung der Kraftaufnehmer zu vermeiden. Selbst wenn eine S9M nur bei $20 \%$ der Nennkraft eingesetzt wird, ist die erreichbare Genauigkeit unter fast allen Umständen hoch genug

- Das Anwendungsspektrum kann erweitert werden: Es ist möglich, ohne den Wechsel des Sensors verschiedene Messaufgaben zu bewältigen, wodurch sich Zeit und Aufwand sparen lassen.

Eine weitere Betrachtung ist in Bild unten. Auf der $X$ - Achse ist eine zu messende Kraft aufgetragen, die zu einer Qualitätskontrolle dienen mag. Auf der Y-Achse wird die Anzahl der produzierten Bauteile gezeigt. Die Streuung der produzierten Teile verteilt sich nach den Gesetzmäßigkeiten der Gaußschen Glockenkurve. Auf den Diagrammen sind jeweils grüne Linien eingetragen, die die erlaubten Toleranzen zeigen, rechts und links dieser Grenzen in rot ist die Messunsicherheit der Kraftmesskette abzulesen.
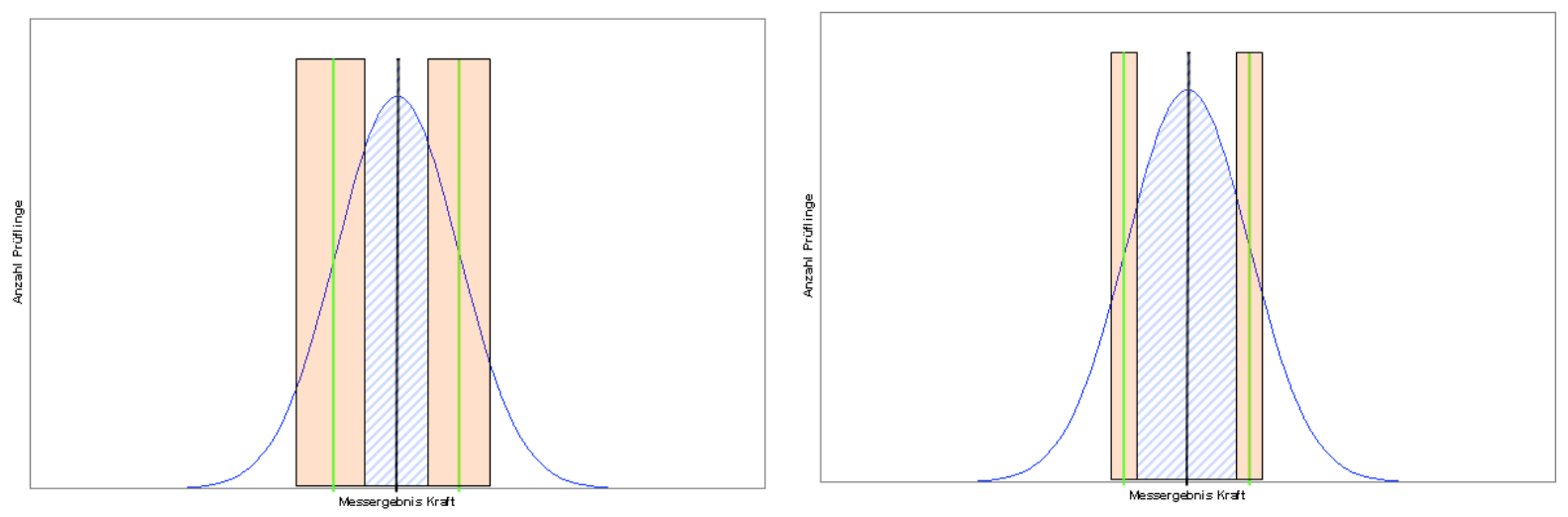

Bild 3 Überwachung eines Prozesses mit einem Kraftmesssystem niedriger (links) und hoher (rechts) Genauigkeit

Zur Beurteilung des Prozesses ist es erforderlich, die Messgenauigkeit des Aufnehmers abzuschätzen. Um eine Gut / Schlecht - Beurteilung durchzuführen, dürfen nur die Bauteile als o.k. gewertet werden, die innerhalb des Sollbereiches abzüglich der Messtoleranz leigen, in den Diagrammen durch die Blau schraffierte Linie symbolisiert. Wie zu erkennen, steigt die Anzahl der tolerierbaren Teile an, wenn die Messgenauigkeit steigt- anders ausgedrückt ist die Anzahl der zu verwerfenden Teile auch von der Messgenauigkeit der Kraftmesskette abhängig.

\section{Zusammenfassung}

Moderne Kraftaufnehmer wie die S9M oder HBM's U10M erreichen vor allem bei den endwertbezogenen Einflussgrößen auf die Messunsicherheit Genauigkeiten über den Klassenstandard hinaus. Neben der Möglichkeit, eine Messkette durch Betrieb im Teillastbereich einzusetzen und dadurch die Toleranz gegen Überlasten wesentlich zu Erhöhung. Hierdurch ist kann eine verbesserte Zuverlässigkeit erreicht werden. Vor allem der minimale Einfluss der Temperatur auf den Nullpunkt erlaubt es, Ein und den 
selben Sensor für verschiedenen Messbereiche zu verwenden, oder mit der hohen Präzision den Anteil der Gutteile zu erhöhen. 